\title{
New Drugs for Scar Treatment
}

Sun Hyung Kwon, Jagannath Padmanabhan, Dominic Henn, Kellen Chen, and Geoffrey C. Gurtner

\section{Contents}

\subsection{Background -458}

52.2 Objectives of the Proposed Chapter -458

52.3 Description of the State-of-the-Art Historical Evolution:

Recent Data - 458

52.4 Transforming Growth Factor- $\beta$ (TGF- $\beta$ ) -459

52.5 Interleukins (IL) $-\mathbf{4 6 0}$

52.6 Mechanotransduction Pathway Inhibitors - 460

52.7 Supportive Articles in the EBM Literature - 461

52.8 Clinical Relevance $-\mathbf{4 6 2}$

52.9 Conclusion -462

References -463 


\subsection{Background}

Cutaneous wound healing following injury is a complex biological process involving the orchestration of the immune system, inflammatory pathways, mechanotransduction pathways, and various types of participating cells. In adult humans, the wound repair process results in the replacement of the damaged functional tissue with a patch of cells (i.e., mostly fibroblasts) and disorganized collagen-rich extracellular matrix that is commonly defined as a scar [1]. While scarring occurs in almost all tissues, it is most apparent in the skin. Cutaneous scarring poses a significant psychological and physiological burden on patients, and an estimated $\$ 12$ billion are spent annually on scar treatments in the USA alone [2]. Additionally, dysfunctional healing and hypertrophic scarring often causes lifelong disability, which has a significant additional economic impact $[1$, 2]. Over 40 million patients undergo surgical procedures annually, which often lead to the formation of hypertrophic scars (HTSs) resulting in substantial morbidity and disfigurement of the skin. Similarly, scar formation occurring as a result of burns and other forms of trauma leads to severe functional disabilities costing the economy over $\$ 4$ billion per year [2]. Keloidal scars, which are characterized by excessive fibrosis in areas of the skin with acne or other minor injuries, represent another major fibrotic skin disorder. Several other skin fibrotic diseases such as Dupuytren's disease, psoriasis, and scleroderma lead to cutaneous scarring as well [2].

In current practice, cutaneous scars are treated using corticosteroid injections or cryotherapy, surgical revisions, topical silicone gels, sheets and pressure garments, radiation, and laser treatments [3]. These traditional treatment modalities have shown mixed success and most methods lead only to partial alleviation of scar formation. Further, many of these treatment modalities are associated with other practical issues such as patient discomfort and pain, multiple and frequent visits to the doctor's office, or even higher recurrence of scars [3]. Recent progress in the identification of key signaling pathways involved in fibrosis and scar formation has led to the emergence of new therapeutic agents for scar treatment, with promising results in animal studies. Agents targeting collagen synthesis pathways, cytokines, growth factors, and cytotoxic agents are under investigation for scar therapy. Of these, drugs that inhibit expression of transforming growth factor (TGF)- $\beta 1 / \mathrm{TGF}-\beta 2$ or augment TGF- $\beta 3$ had shown initial promise, but have been shown to be ineffective in later clinical trials as discussed below [3, 4]. Similarly, treatment of scars with interleukin-10 (IL-10), an interleukin that is involved in regulating inflammatory pathways, has shown some promise in treating psoriasis, but has failed to show any significant benefit for other types of scars [5].
A paradigm shift in our understanding of the scar pathophysiology emerged when the role of mechanotransduction in fibrosis was explored. The role of mechanical stress in scar formation had been described as early as in the 1800s, with the description of Langer's lines of skin tension used for surgical incisions to minimize scarring. But recent work elucidating the role of mechanical cues activating inflammatory pathways and scar-promoting cells highlighted mechanotransduction as a central focus of scar pathophysiology [6]. In the past decade, aberrant activation of mechanosensitive skin cells have been shown to underlie abnormal wound healing and scar formation in various skin diseases including hypertrophic and keloidal scars, Dupuytren's disease, and scleroderma [6]. Offloading of mechanical forces during healing of human skin incisions following abdominal surgeries has been shown to be effective in reducing scar formation in Phase I clinical trials [7]. In particular, focal adhesion kinase (FAK), a molecular mediator of mechanotransduction, has been identified to play a key role in fibrosis and scar formation (- Fig. 52.1) [8]. Animal studies have shown that pharmacological inhibition of FAK can lead to significantly reduced scar formation and accelerated wound healing [9].

In this chapter, we will review the latest pharmacological therapies that are being evaluated for scar management in the currently available literature. This includes agents that have recently completed clinical trials and drugs that target cellular mechanotransduction pathways.

\subsection{Objectives of the Proposed Chapter}

This chapter describes the evidence-based new drug therapies introduced in recent scientific literature for scar mitigation and prevention. Although the underlying pathophysiology of hypertrophic scar formation following injury is far from completely understood, over the past decade, advances in our understanding of the cellular and molecular mechanisms by which fibrotic scars develop have paved the way for devising new approaches in scar therapies.

\subsection{Description of the State-of-the-Art Historical Evolution: Recent Data}

Traditional scar revision technologies include surgical treatment, topical silicone gels, sheets and pressure garments, radiation, and laser treatments [3]. In current practice, intralesional corticosteroidal therapies and antiproliferative 5-fluorouracil therapy are also commonly used to pharmacologically mitigate hypertrophic scars, although their effectiveness is mainly supported 
- Fig. 52.1 Focal adhesion kinase (FAK)-mediated mechanotransduction. Mechanical forces activate FAK, which activates several downstream effectors and transcriptional factors that mediate cellular mechanotransduction [6]. Source: Mechanobiology in Health and Disease, 1st Edition. Elsevier. (C) All rights reserved

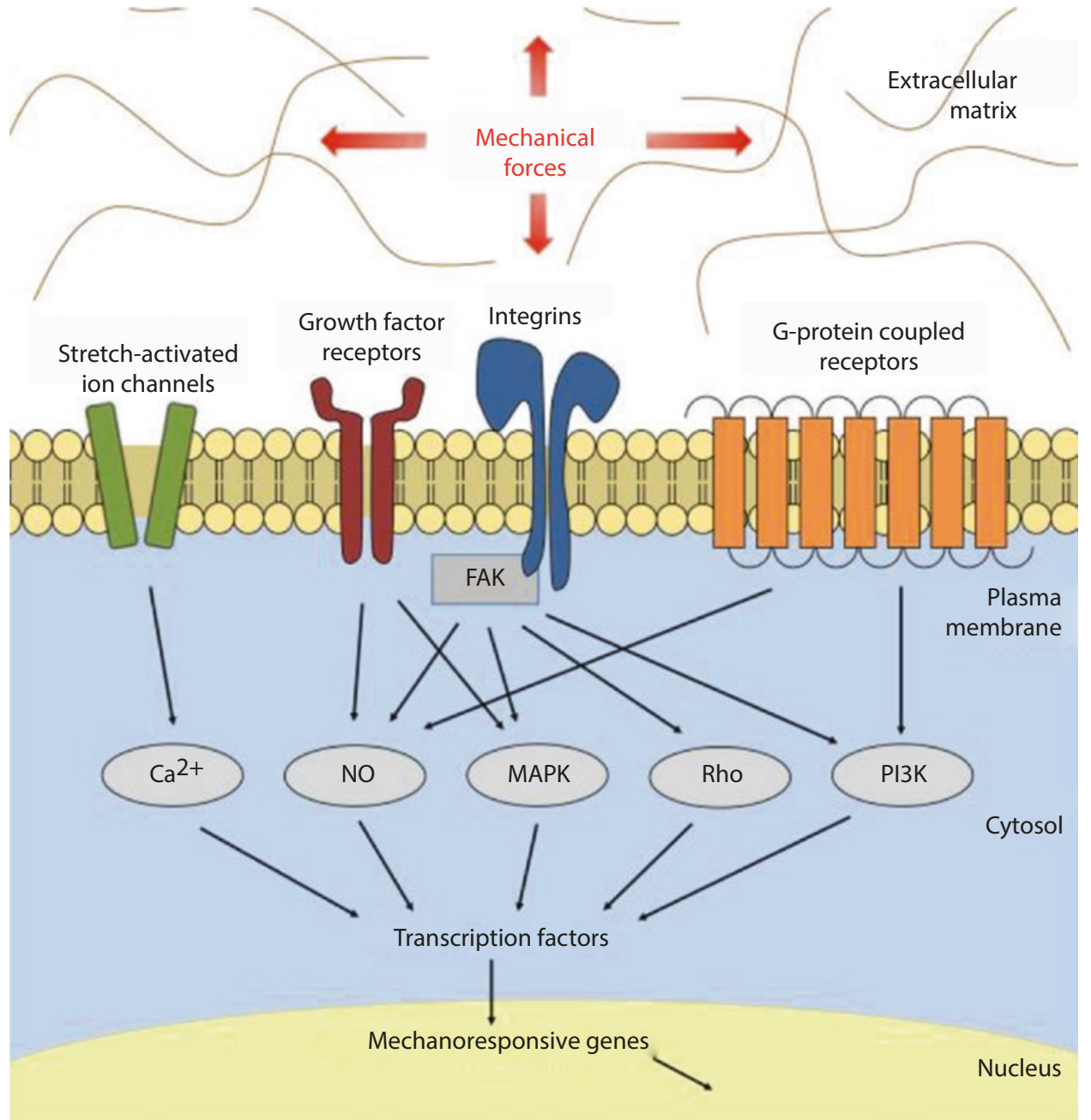

by case studies and preliminary clinical studies [3]. These treatments are often associated with adverse reactions, such as dermal atrophy or hypopigmentation, leading many patients to seek alternative treatment modalities after prolonged use. Recent studies have investigated new pathways to reveal drug targets such as modulators of wound inflammatory cytokines, growth factors, and mechanomodulatory mediators as potential targets for scar-mitigating therapies [6].

\subsection{Transforming Growth Factor- $\beta$ (TGF- $\beta$ )}

TGF- $\beta$ expression is involved in almost all stages of the wound-healing process, including wound inflammation, angiogenesis, extracellular matrix synthesis, and tissue remodeling. Various studies have established the important role this wound-growth factor plays in dermal fibroblast differentiation into myofibroblasts and subsequent scar formation across a range of fibrotic diseases [3, 4]. Studies exploring the scarless and regenerative ability of the fetus have pointed toward the possibility that dif- ferent isoforms of TGF- $\beta$ could contribute to different stages of wound healing. While TGF- $\beta 1$ and TGF- $\beta 2$ are expressed highly in postnatal adults, TGF- $\beta 3$ is the dominant isoform in fetal wound healing, leading many researchers to identify either TGF- $\beta 3$ as a therapeutic target to upregulate or TGF- $\beta 1 / \mathrm{GF}-\beta 2$ as targets to inhibit for scarless wound healing $[3,4]$.

Despite strong preclinical outcomes, current TGF- $\beta$ clinical trials have had disappointing results. Juvista, a new recombinant TGF- $\beta 3$ product developed by Renovo, had shown promise in early phase efficacy trials but failed to meet primary endpoints in Phase III trials $[3,4]$. Renovo also pursued a trial with Juvidex (mannose-6 phosphate), an inhibitor of TGF- $\beta 1 /$ TGF$\beta 2$ [3]. Juvidex failed to meet its primary goal in a Phase II trial but it did meet a number of secondary endpoints [3]. Other researchers have also explored the clinical potential for a recombinant human antibody to neutralize TGF- $\beta 1$ to combat systemic sclerosis, but their clinical studies did not prove any difference in efficacy from control during their Phase I/II trials [3]. TGF- $\beta$ therapy could potentially have confounding results due to its 
dual importance in both normal wound healing and also in excessive fibro-proliferation during scar formation. TGF- $\beta$ receptors are upregulated during fibrosis, and while blocking TGF- $\beta$ expression seems to prevent fibrosis, it can also lead to chronic, nonhealing wounds. Albeit, TGF- $\beta$ continues to be an attractive pharmacological target for a wide variety of fibrotic diseases, and newer strategies modulating TGF- $\beta$ pathophysiology await further investigation.

\subsection{Interleukins (IL)}

Neutrophils and macrophages, two of the major cell types during the inflammatory phase of wound healing, secrete cytokines such as interleukins (ILs). IL-10 has been shown to regulate inflammatory cell function during wound healing by sequestering pro-inflammatory IL- 6 or IL- 8 and by regulating the Th1 cell cytokine production $[3,5]$, leading some research groups to explore the therapeutic effect of administering IL-10 to the wound bed during early wound healing. The previously mentioned Renovo also demonstrated the ability of IL-10 to potentially improve scar healing in human patients during Phase I/II trials administering Prevascar, their recombinant human IL-10 (rhIL-10) product [3]. However, they were unable to pursue Phase III trials after failing the aforementioned Juvidex and Juvista trials. Treatment with rhIL-10 has also been explored in several other clinical trials to potentially combat various inflammatory diseases such as Crohn's diseases and rheumatoid arthritis with disappointing results, but has shown promise in treating psoriasis.

\subsection{Mechanotransduction Pathway Inhibitors}

During wound healing, mechanical stress plays an essential role in promoting pro-fibrotic cellular events through mechanisms that stimulate inflammatory pathway components leading to exuberant fibro-proliferation. Manipulation of the wound mechanoenvironment with new medical devices that can modulate local biomechanics, therefore, has gained a rapidly growing market for scar reduction of surgical wounds [6-8]. Based on long-standing surgical principles clinically used to minimize scar development, these polymer-based medical devices offload mechanical force to release tension imposed upon healing surgical incisions. In particular, incisions created at high-tension body locations such as the central chest, shoulders, knees, ankles, and/or the back are prone to forming HTS than other body sites. For example, abdominoplasty wounds can develop into wide scars due to their natural high-tension closure, and application of a stress-shielding device on these wounds has demonstrated clear efficacy in mitigating scar formation [6]. This technology successfully led polymer stress-shielding devices to the market, and numerous patients have seen benefits in the clinic.

Despite success on surgical incisions, polymer mechanomodulatory devices are difficult to use on largesized excisional wounds, burn injuries, and wounds that formed in contoured body areas. Alternatively, noninvasive therapeutics that pharmacologically target key mechanotransduction (cellular machinery that transduces mechanical stimuli to biochemical signals) pathways have also received highlight in recent literature. Currently, a prototype of such therapeutic agents is at the early preclinical development stage with translational potential.

Small molecule-mediated suppressors of pivotal mechanomodulatory proteins have been studied as anticancer agents for many decades. A non-receptor protein tyrosine kinase, focal adhesion kinase (FAK), is a key upstream mediator of the Integrin mechanotransduction pathway and an important inducer of cell adhesion, proliferation, migration, and angiogenesis $[8,9]$. FAK is known to be deregulated in cancer and is thought to be a rational target to block tumorigenic activities using pharmacological inhibitors [10]. Because emerging studies have shown that FAK transduces mechanical stress signals to stimulate the activation of the FAKERK-MCP-1 signaling pathway and is an important regulator of cancer-promoting pathways [6-8], many pharmaceutical companies have taken effort to develop later-generation FAK inhibitors that display improved pharmacodynamic and pharmacokinetic properties. Clinical trials are ongoing in human cancer subjects, and the therapeutic potential to antagonize stromal solid tumors seems promising to date [10].

In currently available literature, small moleculemediated inhibition of the kinase activity of FAK has been successfully used to prevent experimental bleomycin-induced lung fibrosis and mechanically induced skin HTS formation [6, 9]. FAK inhibition significantly reduced scar-forming fibroblast migration, myofibroblast production of $\alpha$-smooth muscle actin, and aberrant collagen extracellular matrix deposition in these studies (- Fig. 52.2) [9]. Because wound healing and cancer development share similarities in terms of overlapping mechanisms that promote transformation, proliferation, and survival of cells, FAK inhibitor therapy that can be safely delivered to wound sites would be a new and promising approach to wound and scar management. In most circumstances, cutaneous scar development is a localized event; therefore, this provides rationale and support to develop targeted FAK inhibitor delivery modalities in attenuating scar formation. Localized drug delivery has the advantage of circum- 

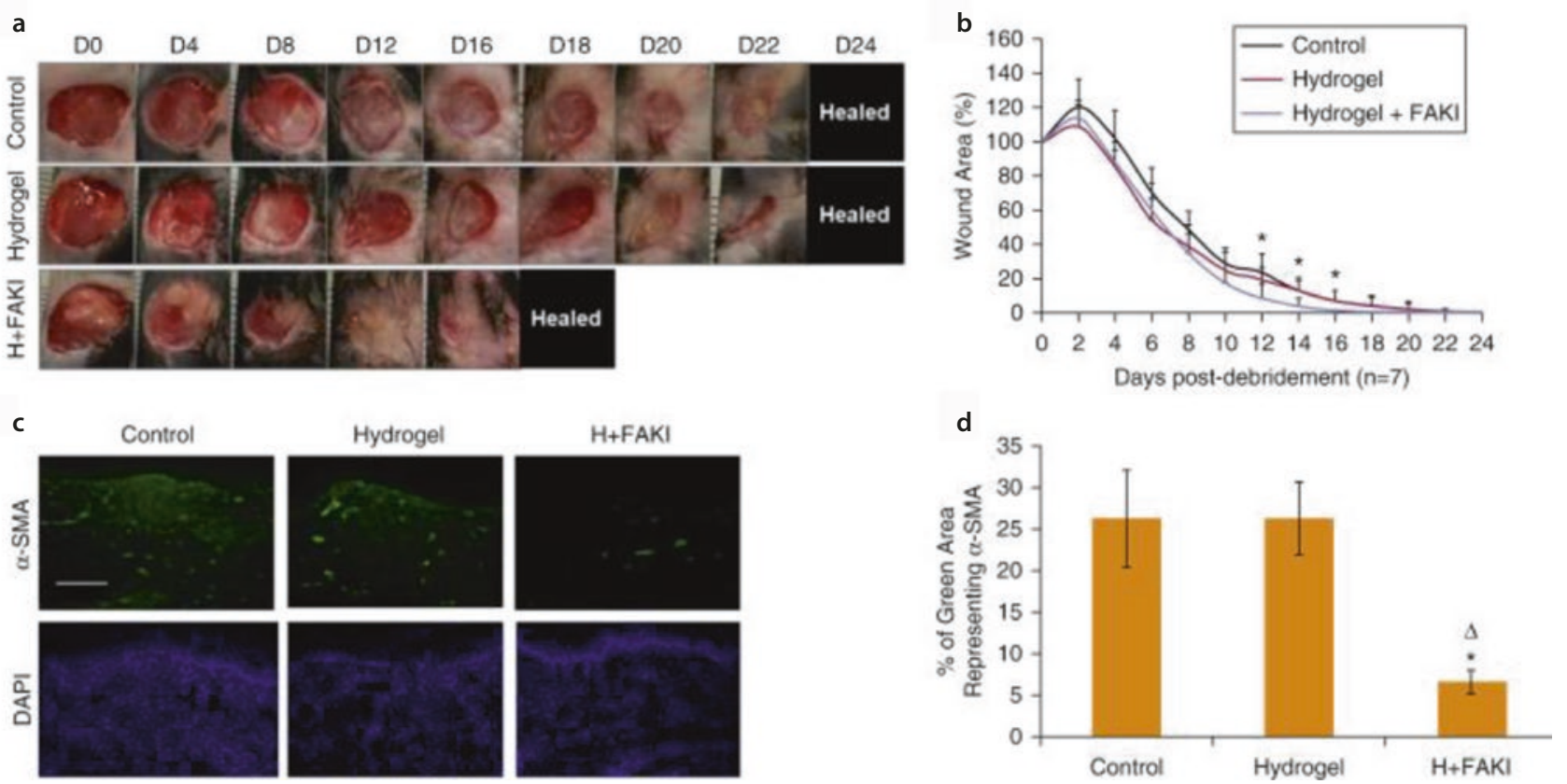

- Fig. 52.2 Pharmacological blockade of focal adhesion kinase (FAK) improves wound healing and reduces scarring of murine burn wounds. (a, b) Mouse contact burn wound healing was accelerated with FAK-I hydrogel treatment. (c, d) Wound expression of

$\alpha$-smooth muscle actin (SMA) was significantly reduced with FAK-I hydrogel treatment [9]. Source: Journal of Investigative Dermatology (2018), Volume 138. Elsevier. (C) All rights reserved

venting systemic toxicity while maximizing bioavailability and local drug efficiency. Localized topical FAK inhibitor therapy ideally requires drug delivery vehicles that are biocompatible, eliciting no or low immunogenicity. Medicated self-adhesive patches that release therapeutic molecules upon dermal contact may not be suitable for large-sized open wounds, and other topical formulations in the form of topical cream, lotion, or ointment are easily subject to over- or under-dosing if application amount per treatment is not accurately measured. The latter is also problematic especially for therapeutic compounds with potential toxicological effects at the systemic level. Therefore, it is scientifically reasonable to devise a dermal delivery vehicle that can deliver topical FAK inhibitor to wounds and/or scars in a controlled manner. Biopolymers have strong advantages as drug delivery carriers because of their ability to carry bioactive compounds to target tissues and cells and to release compounds over a prolonged duration. Recent studies have used collagen-based bioscaffolds to develop biodegradable hydrogels that release FAK inhibitors in a regulated manner upon direct contact with the skin, which has proven preclinical efficacy in rodent wound models for HTS reduction [9]. Development of relevant drug delivery systems and extensive safety studies on potential adverse effects of each agent will be imperative to successful development of pharmaceutical therapies against fibrotic scar formation.

\subsection{Supportive Articles in the EBM Literature}

To date, currently available scar treatment modalities, especially for large-sized excisions and burn-related injuries, have not led to successful clinical outcomes, and the future market for new wound healing and scar mitigation therapies remains strongly promising. Recent progress in the identification of new signaling pathways and cellular components implicated in fibrotic scar formation has advanced our knowledge toward developing nontraditional therapies for scar treatment. Promising results in preclinical studies have resulted in many publications from multiple research groups with some therapies already completing human clinical trials. Scientific rationale for these newer therapies is strong; however, clinical development of some of these agents have faced failure during clinical trial phases. TGF- $\beta$-based therapies, Juvista and Juvidex, both displayed strong preclinical effects but failed during multiple human trials. IL-10 products also showed disappointing clinical outcomes. A mechanomodulatory agent, specifically localized FAK inhibitor therapy, is in its early preclinical development stage with promising animal data recently published. The more we understand the cellular and molecular mechanisms underlying fibrosis and scar formation, the more therapeutic opportunities will be revealed for scar mitigation. 


\subsection{Clinical Relevance}

Cutaneous scar development of the skin, particularly the formation of HTS and keloidal scars following wound healing, constitutes a major clinical challenge for health care, both in terms of individual suffering and economic burden.

Scar formation can occur at any site of dermal lesion resulting from burn-related trauma, or surgical wounds. Burn patients are particularly prone to severe disfigurement and functional impairment through often extensive scarring, and affected children are the most vulnerable patient group in regard to long-term suffering. HTS formation affects a variety of life quality determinants. Cosmetic disfigurement by scar appearance may strongly diminish a person's self-consciousness, as well as their ability to participate in social activities and professional life, and is often associated with serious physical impairments, especially in the context of scarinduced contractures. Moreover, scars frequently cause secondary illnesses requiring complex multidisciplinary treatment not only by plastic surgeons but also by dermatologists, physical therapists, and psychotherapists. In children, abnormal scars can profoundly limit the potential of physical as well as emotional and psychosocial development during childhood. Therefore, effective scar treatment approaches can critically determine a person's long-term ability to lead a self-determined life.

From the perspective of health care professionals, researchers, and health economists, and for the society as a whole, scar formation is perhaps still an underestimated yet growing burden for the public health. In the United States alone, about 500,000 patients are treated for burn injuries each year, resulting in expenses of about $\$ 7.5$ billion. Conversely, treatment of scarring is a considerable economic factor, generating a $\$ 12$ billion annual market with further potential to grow $[1,2,6]$.

It is a long-standing clinical observation that the proneness to HTS formation and keloids is considerably influenced by individual genetic traits as well as ethnic risk profiles [6]. Studies showed that about $16 \%$ of patients with Hispanic or African ancestry are affected from keloidal scarring, as patients with darker skin color are approximately 15 times more likely to be affected than light-skinned individuals. As the individual recurrence risk of keloids also strongly depends on constitutional determinants, progress in research toward their identification and, ultimately, targeted modulation can immediately affect therapeutic strategies of scar formation in the future. Consequently, scarring is a promising as well as challenging field of translational research into personalized medicine in the field of plastic and reconstructive surgery. Beyond that, the search for novel pharmacological treatments addressing molecular targets in the pathways of wound healing and fibrosis has the potential to improve the lives of numerous patients, and also to generate new products for health care of major economic value.

\subsection{Conclusion}

Wound healing and repair following injury is a complex and poorly understood process that leads to cutaneous scar formation, which constitutes a significant health care burden. Despite spending billions of dollars every year on scar management, traditional scar treatment modalities have not proved to be effective. Treatment modalities such as corticosteroid injections and radiation can be largely uncomfortable for the patients. Surgical revisions, which are targeted at reducing the wide base of the scar, are associated with high recurrence rates in the absence of other therapeutic strategies. Identification of key signaling pathways that are dysregulated during abnormal wound healing has led to the emergence of a range of new pharmacological interventions that target growth factors, cytokines, and collagen biosynthesis, and have showed promising results initially but have not led to successful outcomes in later clinical trials.

The clarification of the role of mechanotransduction in fibrosis has opened up a new avenue for scar therapy research. Offloading of mechanical forces of surgical incisions during healing has been shown to be effective in reducing scar formation, which further underscores the importance of mechanical signaling in scar development. Specifically, inhibition of focal adhesion kinase, a key regulator of mechanotransduction has been shown to be effective in attenuating scars and accelerating wound healing. Small molecule inhibition of FAK blocks the activation of inflammatory signaling pathways, thus uncoupling mechanical force from pathologic scar formation. This strategy has been employed to reduce scar formation in burn wounds as well as excisional wounds in mice. Taken together, recent findings indicate that molecular strategies targeting mechanotransduction pathways in general and specifically FAK pathway can effectively reduce fibrosis and show promise as an effective and versatile strategy for scar management. 


\section{Take-Home Messages}

- The underlying pathophysiology of cutaneous hypertrophic scar formation following injury is incompletely understood; however, improved understanding of the molecular mechanisms by which fibrotic scars develop has paved the way for devising new approaches in scar therapies.

- Traditional scar management modalities include surgical revision, corticosteroid injections, and radiation treatment methods. These conventional therapies are often associated with practical issues and higher recurrence rates.

- Recent scientific evidence suggests that mechanical stress plays a crucial role in promoting pro-fibrotic cellular events via pro-inflammatory pathways leading to exuberant cutaneous scar formation and fibrosis.

- New medical devices that can modulate local biomechanics has gained a rapidly growing market for scar reduction of surgical wounds.

- Pharmacological inhibitors of the mechanotransduction pathways are promising evidence-based new scar therapies that block inflammation and pathologic scar formation.

\section{References}

1. Gurtner GC, Werner S, Barrandon Y, Longaker MT. Wound repair and regeneration. Nature. 2008;453:314-21.

2. Padnnanabhan J, et al. In vivo models for the study of fibrosis. Adv Wound Care. 2019; https://doi.org/10.1089/wound.2018.0909.

3. Meier K, Nanney LB. Emerging new drugs for scar reduction. Expert Opin Emerg Drugs. 2006;11:39-47.

4. So K, et al. Avotermin for scar improvement following scar revision surgery: a randomized, double-blind, within-patient, placebo-controlled, phase II clinical trial. Plast Reconstr Surg. 2011;128:163-72.

5. Asadullah K, Sterry W, Volk HD. Interleukin-10 therapy-review of a new approach. Pharmacol Rev. 2003;55:241-69.

6. Kwon SH, Padmanabhan J, Gurtner GC. Chapter 14 - Mechanobiology of skin diseases and wound healing, Editor(s): Stefaan W. Verbruggen, Mechanobiology in Health and Disease, Academic Press, Cambridge, MA. 2018;415-448.

7. Gurtner GC, et al. Improving cutaneous scar formation by controlling the mechanical environment: large animal and phase I studies. Ann Surg. 2011;254:217-2250.

8. Wong VW, et al. Focal adhesion kinase links mechanical force to skin fibrosis via inflammatory signaling. Nat Med. 2011;18:148-52.

9. $\mathrm{Ma} \mathrm{K}$, et al. Controlled delivery of a focal adhesion kinase inhibitor results in accelerated wound closure with decreased scar formation. J Invest Dermatol. 2018;138:2452-60.

10. Infante JR, et al. Safety, pharmacokinetic, and pharmacodynamic phase I dose-escalation trial of PF-00562271, an inhibitor of focal adhesion kinase, in advanced solid tumors. J Clin Oncol. 2012;30:1527-33.

Open Access This chapter is licensed under the terms of the Creative Commons Attribution 4.0 International License (http://creativecommons. org/licenses/by/4.0/), which permits use, sharing, adaptation, distribution and reproduction in any medium or format, as long as you give appropriate credit to the original author(s) and the source, provide a link to the Creative Commons license and indicate if changes were made.

The images or other third party material in this chapter are included in the chapter's Creative Commons license, unless indicated otherwise in a credit line to the material. If material is not included in the chapter's Creative Commons license and your intended use is not permitted by statutory regulation or exceeds the permitted use, you will need to obtain permission directly from the copyright holder.

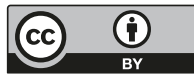

